\title{
OBSERVATIONS OF THE PECULIAR EMISSION-LINE OBJECTS WITH INFRARED EXCESS HD 45677, HD 87643 AND GG CARINAE
}

\author{
JEAN-PIERRE SWINGS \\ Institut d'Astrophysique, B-4200, Cointe-Ougrée, Belgium
}

\begin{abstract}
Spectroscopic data in the visual region is described for the peculiar emission-line objects with IR-excess HD 45677, HD 87643, and GG Car. A model consisting of a central star surrounded by an equatorial disc and a dust shell is suggested.
\end{abstract}

The text included in the present proceedings is based essentially on excerpts from two recent publications by the author:

(i) 'Spectrographic Observations of the Peculiar Be Star with Infrared Excess HD 45677', Astron. Astrophys. 26 (1973), 443.

(ii) 'Similarities in the Spectra of Three Southern Peculiar Emission Line Stars with Infrared Excess: HD 45677, HD 87643 and GG Carinae (HD 94878)', Astron. Astrophys. 34 (1974), 333.

\section{Introduction}

Before beginning the spectral investigations of some peculiar emission line stars with infrared excess, the following preliminary comments ought to be made:

(i) Since we have been hearing about infrared excesses, I wish to illustrate what is an infrared excess on the basis of the energy curve for the B2 IV ep star HD 45677 (Swings and Allen, 1971). In the visible the curve resembles that of a black body $(T \simeq 22000 \mathrm{~K})$, then, instead of dropping to zero in the infrared, it rises abruptly longward of $1.5 \mu$, peaks around $5 \mu$, and remains fairly flat until about $20 \mu$. There is actually 4 times as much energy emitted in the IR as in the visible. Similar curves illustrative of other Bep stars are given in Allen (1973). Following Swings and Allen (1971), it is believed that the IR excess is due to the presence of a circumstellar dust shell around the object, and not to a cool companion.

(ii) One can plot the IR magnitudes on a $H-K$ vs $\mathrm{K}-\mathrm{L}$ colour diagram $(H=1.6 \mu$; $K=2.2 \mu ; L=3.5 \mu)$. One then sees that the objects with strong IR excess are characterized by $\mathrm{H}-\mathrm{K}>1 \mathrm{mag}$. and cluster close to a 'black body line' on which temperatures for the dust are between $1500 \mathrm{~K}$ and 500 or $600 \mathrm{~K}$. Such a diagram, frequently found in papers dealing with IR excess objects is described, e.g., in Allen (1973).

(iii) For early-type stars there exists a strong correlation between the existence of a strong IR excess (H-K $>1$ mag.) and the presence in the spectrum of those peculiar emission-line objects of low excitation forbidden emissions (mainly: [O I], [S II], [Fe II]).

(iv) Some of the objects exhibiting these characteristics are stellar (example HD 45677 , around which exists a rotating equatorial ring formed by ejected matter, as we shall see in a few moments), or star-like (compact $\mathbf{H}$ II regions, like some Henize objects), or look like a pregnant star (He 2-446), or more closely resemble a planetary 
nebula (such as M2-9). In the latter there is evidence that the densities are about 1000 times higher than in normal planetaries.

We tend to believe that the objects mentioned here above, in which variations are observed, might be intermediate between Be stars and planetary nebulae.

\section{The Prototype: HD 45677}

We first consider the prototype of the 'Peculiar Emission Line Objects', i.e. HD 45677 (B2 IV ep; $m_{v} \simeq 8-8.5$ ). On the basis of data in the visible (3100 to $8700 \AA$ ), the following spectrographic information is gathered:

(a) the forbidden emission lines ([O I $],[\mathrm{S} \mathrm{II}],[\mathrm{Fe} \mathrm{II}],[\mathrm{Ni} \mathrm{II}],[\mathrm{Cr} \mathrm{II}], \ldots$ ) are single and sharp;

(b) the double emission lines of permitted Fe II seem to originate in a thin equatorial ring well separated from the stellar photosphere;

(c) the Ti II, Mn II and Cr II lines appear as very broad (or double) emissions with a central sharp absorption (shell or thick ring); the Fe II profiles were similar to those of the metallic lines in the 1940's but have evolved differently since;

(d) the stellar Mg II absorption line $\lambda 4481$ and the circumstellar $\mathrm{Ca}$ II $\mathrm{K}$ line are shown to be variable;

(e) the complex structure of $\mathrm{H} \gamma$ and $\mathrm{H} \delta$ (P Cygni with several emission and absorption components over a wide shallow absorption) varies from night to night;

(f) in the course of a single night, the profiles of $\mathrm{H} \gamma$ and $\mathrm{H} \delta$ varied on a time scale of about two hours, one absorption component disappearing completely.

It is a difficult and doubtful matter to construct a physical model of HD 45677 that takes into account the information listed above. We have however attempted to define the zones around this peculiar Be star where there exists preferential absorption or emission of the various elements, as observed in the last few years (in the visible):

(1) At the surface of the stellar photosphere: wide absorptions of $\mathrm{H}$ and $\mathrm{He} \mathrm{I}$, rotational velocity $v \sin i \simeq 200 \mathrm{~km} \mathrm{~s}^{-1}$.

(2) Near the surface: a variable equatorial disk where the emission in $\mathrm{Mg}$ in $\lambda 4481$ originates (velocity shift between emissions: $\approx 200 \mathrm{~km} \mathrm{~s}^{-1}$; central wide absorption: $\left.v^{\prime} \sin i \approx 120-150 \mathrm{~km} \mathrm{~s}^{-1}\right)$.

(3) Farther 'out': regions causing the complex structure of the Balmer lines: continuous outflow of material (P Cygni profiles) and rapid motions (pulsations, random excursions) of 'clouds' of large dimensions (variations in the structure of $\mathrm{H} \gamma$ and $\mathrm{H} \delta$ ).

(4) Around $10 R_{*}$ : equatorial ring with on the inside $\mathrm{Mn}$ II and $\mathrm{Cr}$ II (rotational velocity $\left.\approx 25 \mathrm{~km} \mathrm{~s}^{-1}\right)$ and on the outside Fe II $\left(v_{\text {rot }} \approx 15 \mathrm{~km} \mathrm{~s}^{-1}\right)$.

(5) Outer region, beyond $\sim 10 R_{*}$ extension of the ring into a disk or a tore (with $T_{\mathrm{e}} \approx 4500 \mathrm{~K}, N_{\mathrm{e}} \approx 10^{8} \mathrm{~cm}^{-3}$ ) in which the emissions of [O I], [S II], [Fe II], [Ni II], [Cr II] originate.

(6) A 'dust shell', responsible for the strong infrared excess: The 5- $\mu$ flux from $\mathrm{HD} 45677$ is equal to that from a $640 \mathrm{~K}$ sphere of diameter $32 \mathrm{AU}$ at $1 \mathrm{kpc}$ and is 
therefore consistent with that from an almost optically thick dust shell heated only by the underlying star.

\section{The Spectra of HD 45677, HD 87643 and GG Carinae}

In the spectral region $\lambda 4160-\lambda 4630 \AA$, all three objects exhibit strong $\mathrm{P}$ Cygni profiles for $\mathrm{H} \gamma$ (very strong and complex in HD 87643) and a series of emission lines due mainly to $\mathrm{Fe}$ II and [Fe II]. As in HD 45677 (Swings, 1973) the [Fe II] lines are sharp, whereas the Fe II lines are either diffuse (HD 87643) or double (GG Car). For HD 45677 a dispersion of $3 \AA \mathrm{mm}^{-1}$ was required to separate the two components of the Fe II lines $\left(\Delta \lambda \sim 30 \mathrm{~km} \mathrm{~s}^{-1}\right)$; this is perhaps also the case for HD 87643. In GG Car, on the contrary, the Fe II lines are clearly double on $12 \AA \mathrm{mm}^{-1}$ plates (Swings, 1974): as for HD 45677, no absorption component seems to appear between the two emissions. The thin equatorial ring that is suggested to be rotating around GG Car would have a velocity of 30 to $35 \mathrm{~km} \mathrm{~s}^{-1}$, compared to about $15 \mathrm{~km} \mathrm{~s}^{-1}$ in the case of HD 45677 (Swings, 1973).

\section{References}

Allen, D. A.: 1973, Monthly Notices Roy. Astron. Soc. 161, 145.

Swings, J. P.: 1973, Astron. Astrophys. 26, 443.

Swings, J. P.: 1974, Astron. Astrophys. 34, 333.

Swings, J. P. and Allen, D. A.: 1971, Astrophys. J. 167, L41. 